\title{
Zur Behandlung von wunden Warzen und Mastitiden im Wochenbett.
}

\author{
Von \\ Dr. W. Rubeska, \\ Prolessor an der Hebammensehule in l'ray.
}

Wunde Warzen sind eines der häufigsten und qualvollsten Uebel im Wochenbett. Ihre Bedeutung für die Entstehung von Brustentzündungen ist allgemein bekannt und anerkannt, dennoch werden sie in den Lehrbüchern der Geburtshilfe sehr stiefmütterlich abgehandelt. Auch in Wochenbettstatistiken findet man sie kaum erwähnt oder nur als Ursachen von von den Geschlechtstheilen nicht ausgehenden Störungen des Wochenbetts. Ihre Behandlung wird auch nur gestreift, präcise erschöpfende Vorschriften werden in der Regel nicht gegeben, wohl mit Unrecht, denn das Wohl und Gedeihen des Kindes hängt häufig von der rechtzeitigen Erkenntniss und Behandlung des kleinen Uebels ab. Denn kommt es zu ausgedehnteren Versehwärungen der Warzen oder zu Mastitiden, so muss das Säugen aufgegeben werden, zum grössten Schaden für das Kind.

Die Affection beginnt entweder mit Blutsuffusion oder mit Bläschenbildung auf der Warzenoberfläche, oder als bogenförmiger Riss an der Basis derselben. Geht in den ersteren Fällen die Oberhaut durch das Saugen des Kindes verloren, so entsteht eine Erosion, welche in die Tiefe greifen und sich durch Ansiedlung von Eitermikroben in ein Geschwür verwandeln kann; ebenso kann aus cinem ursprünglich seichten Epidermisriss eine tiefe halbmondförmige oder circuläre Rhagade an der Basis der Warze entstehen. 
As Ursachen sind schlecht entwickelte, wenig rorragende, schlecht fassbare Warzen, zarte Haut derselben und Milchmangel zu crwähnen. Es ist klar, dass, je weniger die Warze vorragt, jo schlechter sie fassbar ist, um so leichter wird sie aus dem Yunde des Kindes entschlüpfen, um so mehr wird sich das letztere anstrengen, sie im Munde zu behalten und um so leichter wird sich die Epidermis in Bläschen abheben und Veranlassung zur Erosion oder zu einer Rhagade geben.

Je weniger Milch die Brust liefert, um so häufiger und länger und um so kräftiger wird das Kind saugen und um so leichter werden Erosionen und Rhagaden entstehen, namentlich wenn dabei noch zarte Haut vorhanden ist.

Manche Frau bekommt in jedem Wochenbett wundo Warzen. Ihro Häufigkeit beträgt fast $40-50$ pCt. aller stillenden Frauen, wie aus folgender Tabelle erhellt.

\begin{tabular}{l|c|c|c}
\hline \multicolumn{1}{c|}{ Autor. } & $\begin{array}{c}\text { Zahl } \\
\text { der } \\
\text { Stillenden. }\end{array}$ & $\begin{array}{c}\text { Zahl } \\
\text { Wunder } \\
\text { Warzen. }\end{array}$ & $\begin{array}{c}\text { Procent } \\
\text { aller } \\
\text { Stillenden. }\end{array}$ \\
\hline k. Winckel in Dresden. . . . & 2300 & 918 & 39,9 \\
Kehrer in Heidelberg . . . . & - & - & 42,5 \\
liehling in Stuttgart . . . . & 3738 & 1757 & 47,0 \\
Eehling in Basel . . . . . & 2875 & 1421 & 49,1 \\
Rubeska in Prag . . . . . & 3152 & 1244 & 39,4
\end{tabular}

Von meinen Fällen waren 42,2 pCt. bei Erst- und 36,2 pCt. bei Mehrgebärenden.

Die grosse Mehrzahl entstand in der ersten Woche des Wochenbetts, nämlich 95 pCt., und davon die Mehrzahl wieder am dritten und vierten Tage, nämlich 61,6 pCt.

Meine Behandlung war antiseptisch. Prophylactisch wurden die Warzen bei Hausschwangeren täglich mit warmem Wasser und Seifo gewaschen, schlecht vorragende mit Milchpumpen vorgezogen und abwechselnd einen Tag mit 60 proc. Alkohol und den anderen mit Glycerin bestrichen. Während des Stillens wurden die Warzen täglich zweimal mit warmem Seifenwasser und 60proe. Alkohol desinficirt.

Sind sehon Excoriationen oder Rhagaden entstanden, so werden ausser diesen Waschungen die Warzen immerwährend mit einem 
Umschlag von 3 proc. Borwasser bedeckt gehalten. Dor Umschlag wird durch ein übergreifendes Stäck Guttaperchapapier feucht erhalten; das Papier klebt an der warmen Brusthaut an und hält den Umschlag an Ort und Stelle. Durch diese Waschungen und durch den antiseptischen Occlusivverband werden die wunden Warzen rein gehalten, es entstehen keine Borken und Krusten auf donselben, welche beim Stillen immer frisch abgerissen werden, dio Eiterkokken, welche auf die Wunden gelangen, werden zum Theil abgetödtet, zum Theil in ihrer Virulenz abgeschwächt, die Wunden bleiben rein, feucht, die Ränder blass, nicht infiltrirt, nicht entzündet, die Schmerzen bleiben geringer und das Stillen kann fortgesetzt werden.

Sind die Wunden ausgedehnter und tiefer, so wird dio Brust nur mit Warzenhütchen geschützt dem Kinde gereicht. Am besten sind die einfachen Gummihütchen, weil bei ihnen das Kind neben der Aspiration auch den Druck der Kiefer auf die Milchsinus unter der Warze in Anwendung ziehen kann, was für das Sauggeschäft nothwendig ist, wie Basch nachgewiesen hat.

Dieses Ausdrücken entfällt bei den Glasgummihütchen von Bailly und bei der Tetérelle biaspiratrice von Auvard, und deshalb trinkt sich das Kind nur bei reichlich und leicht fliessender Milch satt.

Bei dieser Behandlung stillten fast alle Mütter trotz wunder Warzen ihre Kinder weiter. Bei Milchmangel wurde zugefüttert.

Die Mehrzahl der Warzen heilte dabei im Laufe ciner Woche. Folgende Tabelle führt das näher aus.

\begin{tabular}{|c|c|c|c|c|c|c|c|c|c|}
\hline \multirow{2}{*}{$\begin{array}{c}\text { Enl- } \\
\text { stehungs- } \\
\text { tag } \\
\text { wunder } \\
\text { Warzen. }\end{array}$} & \multicolumn{3}{|c|}{ Zahl der Fälle: } & \multicolumn{6}{|c|}{ Gehoilt bis zam 10. Tage: } \\
\hline & 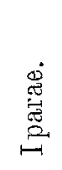 & 焉 & $\begin{array}{l}\text { 离 } \\
\text { 芯 } \\
\text { 岕 } \\
\text { 心 }\end{array}$ & 恣 & 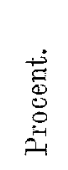 & 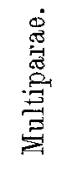 & 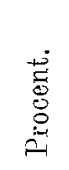 & 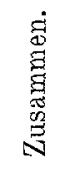 & 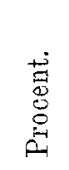 \\
\hline 2. & 69 & 69 & 183 & 34 & 49,0 & 44 & 63,7 & 78 & 56.5 \\
\hline 3. & 241 & 192 & 433 & 155 & 64,3 & 148 & 77,0 & 303 & 70,0 \\
\hline 4. & 181 & 143 & 324 & 127 & 74,0 & 111 & 77,6 & 238 & 73,4 \\
\hline 5. & 111 & 69 & 180 & 87 & 78,3 & 54 & 78,2 & 141 & 78,3 \\
\hline 6. & 43 & 28 & 71 & 31 & 72,0 & 20 & 71,0 & 51 & 71,8 \\
\hline 7. & 18 & 12 & 30 & 13 & 72,2 & 9 & 75,0 & 22 & 73,3 \\
\hline Summa & 663 & 513 & 1176 & 447 & 67,4 & 386 & 75,2 & 833 & 70,8 \\
\hline
\end{tabular}


Aus der Tabelle ist ersichtlich, dass von 1176 in der ersten Woche des Wochenbetts entstandenen wunden Warzen 833 oder 70,8 pCt. bis zum 10. Tage geheilt waren.

Aus derselben erhellt auch, dass, je früher die Warzen wund wurden, um so längere Zeit brauchten sie zur Heilung. Von den am 2. Tage entstandenen heilten bis zum 10. Tage oder im Taufe von 8 Tagen 56,5 pCt., wohingegen von den am 5. Tage ontstandenen bis zu demselben Tage oder im Laufe von 5 Tagen sehon 78,3 pCt.

Der Erfolg scheint mir gut zu sein. Ein Vergleich mit andoren Behandlungsmethoden fehlt, ich habe keine diesbezüglichen Ziffern finden kösnen.

Ein anderer Prüfstein des Werthes einer Behandlung wunder Warzen ist das Verhältniss, wie oft Mastitis nachfolgt.

Es ist eine alte Erfahrung, dass den meisten puerperalen llastitiden wunde Warzen vorausgehen. Diese sind nämlich häufig Sitz von Eiterkokken, namentlich des Staphylococeus pyogenes albus, manchmal auch des aureus, wie ich dureh Culturen in vielen Fällen nachgewiesen habe. Von den Wunden dringen dann die Eiterkokken in die Brust entweder durch die Milchgänge oder dureh die Jymphbahnen und verursachen Mastitis. Je besser also die wunden Warzen versorgt sein werden, um so seltener wird Mastitis hinzukommen.

Wie oft dies geschieht, erhellt aus folgender Tabelle.

\begin{tabular}{|c|c|c|c|c|c|c|}
\hline Antor. & 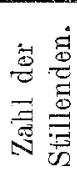 & 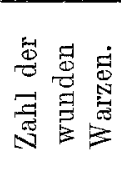 & 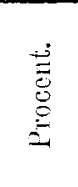 & 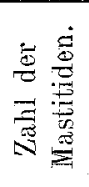 & 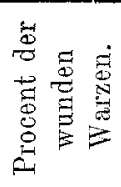 & 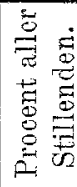 \\
\hline$\therefore$ Winckel in Uresden. & 2300 & 918 & 39,9 & 136 & 14,8 & 4,1 \\
\hline Kehrer in Heidelberg. . & 1600 & 一 & - & 59 & - & 3,6 \\
\hline Fehling in Stuttgart. & 3768 & 1757 & 47,0 & 119 & 6,7 & 3,18 \\
\hline Hehling in Halle ... & 878 & 一 & - & 17 & - & 1,93 \\
\hline Olshausen in Halle. & 972 & $\ldots$ & - & 32 & - & 3,29 \\
\hline Rubestra in Prag . . & 3152 & 1244 & 39,4 & 17 & 1,44 & 0,54 \\
\hline
\end{tabular}

Bei unserer Behandlung wunder Warzen ist also die Zahl der Yastitiden am kleinsten. 
Was den Ausgang der Mastitiden anbelangt, so ist er in der Mehrzahl der Fälle Resorption. Fehling behauptet, dass man Eiterung zu erwarten hat, wenn das Fieber länger als 2 Tage anhält. Er wendet Sublimatumschläge an.

Dabei sah er in Stuttgart unter 119 Mastitiden 23 Eiterungen $=19,3$ pCt., in Halle unter 17 Mastitiden 5 Eiterungen = 29,8 pCt., Olshausen in Hallo unter 32 Fällen 6 Eiterungen $=19,3$ pCt., und ich in Prag unter 17 Mastitiden 2 Fiterungen $=11,7 \mathrm{pCt}$.

Meine Behandlung der beginnenden Mastitis besteht in energischer Desinfection der Warzen, in Umschlägen mit 0,5 proc. Sublimatlösung für 2 Stunden auf die Warze, in der Aspiration der ersten Milch aus den Milchgängen mittelst Milchpumpe, und in der Application einer Eisblase auf die Brust. Beginnt die Mastitis mit einem Schüttelfrost, hohem Fieber und sehr schmerzhafter Infiltration, so mache ich in dieselbe sofort eine parenchymatöse Injection von 3 proc. Carbolwasser, 2-3 Pravazsche Spritzen.

Ist der Beginn weniger stürmisch, so wird diese Injection gomacht, wenn am 2. Tag die Schmerzhaftigkeit und das Fieber nicht zurückgeht. Das kleine Procent unserer vereiterten Mastitiden wäre diesen Injectíonen zu verdanken, obzwar die Zahl derselben noch zu klein ist, um daraus bindende Schlüsse ziehen zu können. Die Injectionen sollen die Vermehrung der Eiterkokken in dem entzündeten Lappen beschränken und dem Organismus den Kampf gegen dieselben erleichtern. Einige Beispiele scheinen schr für die Wirksamkeit dieser Carbolinjectionen zu sprechen.

\section{Fall I.}

24jährige, Ipara bekam am 2. Wochenbettstage Excoriationen der Warzen und hatte vom 4.-8. Tage Fieber bis zu $39,2^{\circ} \mathrm{C}$. mit 104 bis 136 Pulsschlägen. Am 9. Tage bekam sie bei einer Temperatur von $39,9^{\circ}$ eine rechtsseitige Mastitis; am nächsten Tage Vormittags wurden in den entzündeten Brustdrüsenlappen 3 Pravaz'sche Spritzen von 3 proc. Carbolwasser injicirt, Nachmittags war die Temperatur $38,9^{\circ}$, Abends 36,9 , am folgenden (11.) Tage war die Temperatur normal und die Mastitis zertheilte sich.

Fall 11 .

20jährige, Ipara, welche mit einer Gesichtslage spontan niedergekommen ist, bekam am 4 . Wochenbettstage eine Schüttelfrost, Tem- 
peratur $38,5^{\circ}$ und $39^{\circ}$, und rechtsseitige Mastitis. In diese wurden 2 Spritzen Carbolwasser eingespritzt, am nächsten Tage war die Temperatur normal und die Mastitis ging in Resorption über. Am 9. Tage trat bei 38,6, 390 Temperatur linlssseitige Mastitis auf, welche nacb Injection von 2 Spritzen Carbolwasser auch zurückging. Dje Hutter stillte ihr Kind weiter. Nach der Injection wurde das Kind erst nach 4 Stunden an die kranke Brust angelegt.

\section{Fill III.}

23jährige, II para, bekam nach spontaner Geburt ohne innere Untersuchung am (6. Tage elne Temperatur von $38,3,38,20$, am 7 . Tage 37,38 , Hastitis dextra, am 8. 37, $, 37,7$, am 9. Tage 37,38 , am 10. Tage 37,1, 38,1, 390 bei 120 Pulsen und fortbestehender Mastitis. Aus dem dem entzündeten Lappen entsprechenden Milehgange wurde Vlilch ausgedrückt und aus dem Lappen selbst mittelst Pravaz'scher Spritze aspirirt. In beiden Milcharten wurden spärliche Eiterkörperchen und Staphylococcus pyogenes albus nachgewiesen. Am selben Tag wurden in den infiltrirten Lappen 3 Spritzen Carbolwasser eingespritzt, am nächsten 11. Tage war die Temperatur 37,4, 38, am 12. Tage normal, die Schmerzhaftigkeit börte auf zud die Mastitis ging zurück.

\section{IFall IV.}

20jährige, I para, bekam am 3. Wochenbettstage wunde Warzen, am 6. Tage rechtsseitige Mastitis mit einer Temperatur von 38,7 , an 7. Tage 39.9 Vormittags und 39,9 Nachmittags, 4 Spritzen Carbolwasser; an 8. Tage 37,9, 38,7, die Schmerhaftigkeit geringer. In der nächstfolgenden Nacht Frösteln, am 9. Tage 38,8, 39,3, am 10. Tage $38, \overline{0}, 39$, Mastitis auf der anderen Seite. 2 Spritzen Carbolwasser, am 11. Tage Temperatur 37, 38,70, Frösteln, wieder grössere Schmerzhaftigkeit der rechten Brust, neuerdings 2 Spritzen Carbolwasser, die Temperatur am nächsten Tage normal, beide Mastitiden gingen in Resorption über.

\section{Fall V.}

42jährige, II para, bekam am 4. Wochenbettstage wunde Warzen, am 9. Tage einen Schüttelfrost, Temperatur 40,1, 39,4, linksseitige Mastitis im unteren äusseren Octanten. 2 Spritzen Carbolwasser. Am 10. Tag Temperatur 37,1, Nachmittags 38,2 , im oberen äusseren Oetanten derselben Brust ein entzündliches Infiltrat, 2 Spritzen Carlolwasser, am 11. Tag Infiltration im unteren imneren Octanten, wiedcr 2 Spritzen Carbolwasser. Alle Infiltrate gingen zurück, die Mutter stillte ihr Kind weiter.

Aehnlich war das Resultat in weiteren 7 Fällen.

Nur in 2 Fällen kam es trotz parenchymatöser Carbolwasserinjectioneu zur Eiterung. Die Falle waron folgende.

\section{Falı I}

29 jährige, 1 para, bokam am 4. Tage wunde Warzen und am 12. Tage bei einer Temperatur von $39,4,39,3^{0}$ linksseitigo Mastitis. 
Es wurden 2 Spritzen Carbolwasser eingespritzt und als am nächsten Tage die Temperalur $38,8,39^{\circ}$ hoch blieb, weitere 2 Spritzen. Die Temperatur erreichte am folgenden Tage nur noch $37,8^{\circ}$ und blieb dann normal, aber die Infiltration nahm zu, am 17. Tage wurde Fluctuation nachgewiesen und der Abscess eröffnet. Ans dem Eiter wurde Staphylococcus pyogenes aureus gezü.chtet.

\section{Fall II.}

20jährige, Ipara, bekam am 4. Wochenbettstage wunde Warzen, am 7. Tage bei $39^{\circ}$ Temperatur, rechtsseitige Mastitis im oberen inneren Octanten; 2 Spritzen Carbolwasser, am 8. Tage Temperatur $37,7,39,8^{0}, 104$ Pulse, neuerdings 2 Spritzen Carbolwasser.

Die Körperwärme blieb dann durch 3 Tage normal und das Infiltrat verkleinerte sich, aber dann ging die Temperatur wieder in die Höhe und am 14. Tage wurde die vereiterte Mastitis incidirt. Später entstanden in derselben Brust noch weitere 3 Abscesse, so dass sich die Heilung sehr in die Länge zog.

Schädliche Folgen der Carbolinjectionen, Erscheinungen von Carbolintoxication habe ich nie gesehen, die Schmerzen waren nicht gross und hörten bald auf, so dass ich die Carbolinjectionen empfehlen kann.

Was das pathogene Agens der Mastitiden anbelangt, so habe ich unter 16 untersuchten Fällen 9 mal den Staphylococcus pyogenes aureus allein, 3 mal den Staphylococcus pyogenes albus allein, 3 mal diesen gemeinschaftlich mit dem aureus und 1 mal Streptococcus pyogenes nachgewiesen. Dieser Fall betraf eine Schwangere im 8. Nonat, welche seit 3 Tagen an Schmerzen in der rechten Brust litt und daselbst einen Abscess bekam, der incidirt und aus dessen Eiter Streptococcus pyogenes rein gezüchtet wurde. Die Warze war mit einer dicken Schmutzkruste bedeckt, unter derselben die Haut intact. Der Abscess war einfach und heilte rasch zu. 
184 Rubeska, Zar Behandlung von wunden Warzen und Mastitiden etc.

\section{Literatur.}

1. พ. Winckel, Ber, u. Studien aus d. Kgl. sächs. Entbindungsanstalt in Dresden. Bd. I. 1876.

2. Haussmann, Zur Behandlung wunder Brustwarzen. Berl. klin. Wochenschrift. 1878 . No. 14 u. 15.

3. Kaltenbach, Erosionen der Brustwarzen als puerperalo Infectionsstelle. Centralbl. f. Gyn. 1883. o. 5.

4. Kehrer, Entstehung and Prophylaxe der wunden Brustwarzen. Beitr. z. kilin. u. experim. Geburtsk. Bd. II. H. 1.

5. Kehrer, Müller's Handbuch der Geburtshülfe. Bd. III. 1889.

6. Fehling, Die Physiologie und Pathologie des Wochenbettes. II. Auflage. Stuttgart 1897.

7. Basch, Beiträge zur Kenntniss des menschlichen Milchapparats. Arch. f. Gyn. Bd. XLIV. H, 1. 